\title{
Iterative improvement of the shallow-ice approximation
}

\author{
Ondřej SOUČEK, ${ }^{1,2}$ Zdeněk MARTINEC ${ }^{1,3}$ \\ ${ }^{1}$ Department of Geophysics, Charles University in Prague, V Holešovičkách 2, 18000 Praha 8, Czech Republic \\ E-mail: soucek@karel.troja.mff.cuni.cz \\ ${ }^{2}$ Research Institute of Geodesy, Topography and Cartography, 25066 Zdiby 98, Czech Republic \\ ${ }^{3}$ GeoForschungsZentrum Potsdam (GFZ), Telegrafenberg, D-14473 Potsdam, Germany
}

\begin{abstract}
We present a new algorithm for a fast iterative improvement of the shallow-ice approximation (SIA) for the modeling of glacier flow. Based on the traditional SIA scaling assumptions, the solution of the Stokes problem is found by an operator-splitting iterative technique. The SIA solution obtained in the first step is successively improved to obtain a higher-order approximation. Each iterative step has computational demands comparable to solving the SIA, which makes the algorithm substantially faster than other higher-order or full-Stokes solvers. The performance of the algorithm is tested on a model example taken from the ISMIP-HOM intercomparison project.
\end{abstract}

\section{INTRODUCTION}

Increasing demands on the accuracy of solutions for glacier flow and improving computational possibilities are pushing the glaciological community to abandon the traditional shallow-ice approximation (SIA) (Hutter, 1983) and include the computation of longitudinal stresses in numerical models. This is essential when the scaling assumptions of the SIA approach (Greve, 1997) are violated, such as for small alpine glaciers, ice streams, floating ice shelves, groundingline dynamics and other, usually small-scale, examples of ice dynamics.

A number of theoretical and numerical approaches have been proposed and tested, including several higher-order approximations of the Stokes problem by 'multilayer' methods (Blatter, 1995; Pattyn, 2003; Saito and others, 2003). For their classification and discussion see Hindmarsh (2004). Also, a number of exact full-Stokes solvers have been developed, based on various numerical techniques such as finite-difference (e.g. Pattyn, 2003), spectral (Hindmarsh, 2004), finite-volume (Price and others, 2007) and finiteelement methods (Le Meur and others, 2004; Zwinger, 2007; Gagliardini and Zwinger, 2008).

However, making the jump from the SIA approach to more advanced models substantially increases computational demands, which subsequently complicates the embedding of these techniques into large-scale models. In this paper, we present a computational algorithm that provides an approximate solution to the Stokes problem that is more accurate than the SIA solution, but still applies the traditional SIA scaling assumption on the aspect ratio of a glacier. The primary criterion for the construction of the new algorithm is computational efficiency.

\section{THE STOKES PROBLEM FOR FLOW}

Our aim is to solve the boundary-value problem that allows us to model an incompressible Stokes flow with non-linear viscous rheology in a Cartesian geometry.

We are looking for the solution to the Stokes equation with the acceleration term neglected, that is, the linear momentum equation of the form

$$
\operatorname{div} \boldsymbol{\tau}+\rho \vec{g}=\overrightarrow{0},
$$

where $\rho$ is the ice density, $\vec{g}$ is the gravity acceleration at the
Earth's surface and the stress tensor, $\boldsymbol{\tau}$, is given by

$$
\boldsymbol{\tau}=-p \mathbf{I}+\boldsymbol{\sigma},
$$

where $p$ and $\boldsymbol{\sigma}$ are the isotropic and deviatoric parts of $\boldsymbol{\tau}$, respectively, and $\mathbf{I}$ is the identity tensor. Since the ice flow is assumed to be incompressible and ice density homogeneous, the divergence-free constraint on the ice velocity, $\vec{v}$, is to be satisfied:

$$
\operatorname{div} \vec{v}=0 .
$$

A glacier is bounded by two continuously differentiable surfaces

$$
\begin{array}{ll}
x_{3}=f_{\mathrm{s}}\left(x_{1}, x_{2}\right) & \text { (free surface) } \\
x_{3}=f_{\mathrm{b}}\left(x_{1}, x_{2}\right) & \text { (bed), }
\end{array}
$$

where $x_{1}, x_{2}, x_{3}$ are the Cartesian coordinates. Considering the typical horizontal, $[L]$, and vertical, $[H]$, dimensions of a glacier, and typical horizontal, $\left[V_{\leftrightarrow}\right]$, and vertical, $\left[V_{\uparrow}\right]$, velocities of the glacier flow with the aspect ratio

$$
\epsilon=\frac{[H]}{[L]}=\frac{\left[V_{\uparrow}\right]}{\left[V_{\leftrightarrow}\right]}
$$

the following scaling is introduced

$$
\begin{aligned}
\left(x_{1}, x_{2}, x_{3}\right) & =\left([L] \tilde{x}_{1},[L] \tilde{x}_{2},[H] \tilde{x}_{3}\right), \\
\left(v_{1}, v_{2}, v_{3}\right) & =\left(\left[V_{\leftrightarrow}\right] \tilde{v}_{1},\left[V_{\leftrightarrow}\right] \tilde{v}_{2},\left[V_{\uparrow}\right] \tilde{v}_{3}\right), \\
\left(f_{s}\left(x_{1}, x_{2}\right), f_{\mathrm{b}}\left(x_{1}, x_{2}\right)\right) & =[H]\left(\tilde{f}_{\mathrm{s}}\left(\tilde{x}_{1}, \tilde{x}_{2}\right), \tilde{f}_{\mathrm{b}}\left(\tilde{x}_{1}, \tilde{x}_{2}\right)\right),
\end{aligned}
$$

where the scaling of the stress tensor is chosen as

$$
\left(p, \boldsymbol{\sigma}_{i j}\right)=\rho g[H]\left(\tilde{p}, \tilde{\boldsymbol{\sigma}}_{i j}\right) \quad i, j=1,2,3 .
$$

Such a scaling only non-dimensionalizes the stresses, without requiring the scaled quantities to be of the order of unity, in which case a more appropriate scaling would be that suggested by Greve (1997).

The Stokes equation (1) for the scaled quantities reads as

$$
\begin{aligned}
& 0=-\frac{\partial \tilde{p}}{\partial \tilde{x}_{1}} \epsilon+\frac{\partial \tilde{\boldsymbol{\sigma}}_{11}}{\partial \tilde{x}_{1}} \epsilon+\frac{\partial \tilde{\boldsymbol{\sigma}}_{12}}{\partial \tilde{x}_{2}} \epsilon+\frac{\partial \tilde{\boldsymbol{\sigma}}_{13}}{\partial \tilde{x}_{3}}, \\
& 0=-\frac{\partial \tilde{p}}{\partial \tilde{x}_{2}} \epsilon+\frac{\partial \tilde{\boldsymbol{\sigma}}_{12}}{\partial \tilde{x}_{1}} \epsilon+\frac{\partial \tilde{\boldsymbol{\sigma}}_{22}}{\partial \tilde{x}_{2}} \epsilon+\frac{\partial \tilde{\boldsymbol{\sigma}}_{23}}{\partial \tilde{x}_{3}}, \\
& 1=-\frac{\partial \tilde{p}}{\partial \tilde{x}_{3}}+\frac{\partial \tilde{\boldsymbol{\sigma}}_{13}}{\partial \tilde{x}_{1}} \epsilon+\frac{\partial \tilde{\boldsymbol{\sigma}}_{23}}{\partial \tilde{x}_{2}} \epsilon-\frac{\partial\left(\tilde{\boldsymbol{\sigma}}_{11}+\tilde{\boldsymbol{\sigma}}_{22}\right)}{\partial \tilde{x}_{3}},
\end{aligned}
$$


where the symmetry of the deviatoric stresses, $\boldsymbol{\sigma}_{i j}$, was used and $\boldsymbol{\sigma}_{33}$ was eliminated from the system of equations by making use of the trace-free constraint on the deviatoric stresses in such a way that only six independent stress unknowns are considered. The incompressibility condition (3) for scaled quantities then reads as

$$
\frac{\partial \tilde{v}_{1}}{\partial \tilde{x}_{1}}+\frac{\partial \tilde{v}_{2}}{\partial \tilde{x}_{2}}+\frac{\partial \tilde{v}_{3}}{\partial \tilde{x}_{3}}=0
$$

\section{Boundary conditions}

We assume stress-free conditions at the surface, i.e.

$$
\boldsymbol{\tau} \cdot \vec{n}=\overrightarrow{0}, \quad \text { at } \quad x_{3}=f_{\mathrm{s}}\left(x_{1}, x_{2}\right),
$$

where $\vec{n}$ is the unit outer normal. In the scaled form, we have

$$
\begin{aligned}
& 0=\tilde{p}\left(\frac{\partial \tilde{f}_{\mathrm{S}}}{\partial \tilde{x}_{1}}\right) \epsilon-\tilde{\boldsymbol{\sigma}}_{11}\left(\frac{\partial \tilde{f}_{\mathrm{s}}}{\partial \tilde{x}_{1}}\right) \epsilon-\tilde{\boldsymbol{\sigma}}_{12}\left(\frac{\partial \tilde{f}_{\mathrm{s}}}{\partial \tilde{x}_{2}}\right) \epsilon+\tilde{\boldsymbol{\sigma}}_{13} \\
& 0=\tilde{p}\left(\frac{\partial \tilde{f}_{\mathrm{s}}}{\partial \tilde{x}_{2}}\right) \epsilon-\tilde{\boldsymbol{\sigma}}_{12}\left(\frac{\partial \tilde{f}_{\mathrm{s}}}{\partial \tilde{x}_{1}}\right) \epsilon-\tilde{\boldsymbol{\sigma}}_{22}\left(\frac{\partial \tilde{f}_{\mathrm{s}}}{\partial \tilde{x}_{2}}\right) \epsilon+\tilde{\boldsymbol{\sigma}}_{23} \\
& 0=\tilde{p}+\tilde{\boldsymbol{\sigma}}_{11}+\tilde{\boldsymbol{\sigma}}_{22}+\tilde{\boldsymbol{\sigma}}_{13}\left(\frac{\partial \tilde{f}_{\mathrm{s}}}{\partial \tilde{x}_{1}}\right) \epsilon+\tilde{\boldsymbol{\sigma}}_{23}\left(\frac{\partial \tilde{f}_{\mathrm{S}}}{\partial \tilde{x}_{2}}\right) \epsilon
\end{aligned}
$$

at $\tilde{x}_{3}=\tilde{f}_{\mathrm{s}}\left(\tilde{x}_{1}, \tilde{x}_{2}\right)$.

At the glacier bed, we assume no-slip conditions, i.e.

$$
\vec{v}=\overrightarrow{0}, \quad \text { at } \quad x_{3}=f_{b}\left(x_{1}, x_{2}\right)
$$

or, in the scaled form,

$$
\tilde{\vec{v}}=\overrightarrow{0}, \quad \text { at } \quad \tilde{x}_{3}=\tilde{f}_{\mathrm{b}}\left(\tilde{x}_{1}, \tilde{x}_{2}\right)
$$

\section{Rheology}

The connection between the deviatoric stress and the velocity is made by a rheological equation. Here we consider Glen's flow law (e.g. Paterson, 1981) (the Einstein's summation convention is used if not otherwise stated):

$$
\boldsymbol{\sigma}_{i j}=2 \eta \dot{\varepsilon}_{i j,} \quad \eta=\frac{1}{2} A^{-1 / n} \dot{\varepsilon}_{I I}^{(1-n) / n}, \quad n=3,
$$

where $A$ is an ice flow parameter and $\dot{\varepsilon}_{\| l}$ is given by

$$
\dot{\varepsilon}_{l l}=\sqrt{\frac{\dot{\varepsilon}_{i j} \dot{\varepsilon}_{i j}}{2}},
$$

and

$$
\dot{\varepsilon}_{i j}=\frac{1}{2}\left(\frac{\partial v_{i}}{\partial x_{j}}+\frac{\partial v_{j}}{\partial x_{i}}\right) .
$$

Applying the scaling, Equations (7-9), the rheology equation becomes

$$
\begin{aligned}
& \tilde{\boldsymbol{\sigma}}_{13}=\tilde{\eta}\left(\frac{\partial \tilde{v}_{1}}{\partial \tilde{x}_{3}}+\epsilon^{2} \frac{\partial \tilde{v}_{3}}{\partial \tilde{x}_{1}}\right) \\
& \tilde{\boldsymbol{\sigma}}_{23}=\tilde{\eta}\left(\frac{\partial \tilde{v}_{2}}{\partial \tilde{x}_{3}}+\epsilon^{2} \frac{\partial \tilde{v}_{3}}{\partial \tilde{x}_{2}}\right), \\
& \tilde{\boldsymbol{\sigma}}_{12}=\epsilon \tilde{\eta}\left(\frac{\partial \tilde{v}_{1}}{\partial \tilde{x}_{2}}+\frac{\partial \tilde{v}_{2}}{\partial \tilde{x}_{1}}\right), \\
& \tilde{\boldsymbol{\sigma}}_{11}=2 \epsilon \tilde{\eta} \frac{\partial \tilde{v}_{1}}{\partial \tilde{x}_{1}}, \\
& \tilde{\boldsymbol{\sigma}}_{22}=2 \epsilon \tilde{\eta} \frac{\partial \tilde{v}_{2}}{\partial \tilde{x}_{2}}
\end{aligned}
$$

$$
\begin{aligned}
\tilde{\eta}= & \left(2 \epsilon^{-1} C \mathcal{V}\right)^{-\frac{1}{3}}, \\
C= & \frac{A(\rho g)^{3}[H]^{5}}{\left[V_{\leftrightarrow}\right][L]}, \\
\mathcal{V}= & \left(\frac{\partial \tilde{v}_{1}}{\partial \tilde{x}_{3}}+\epsilon^{2} \frac{\partial \tilde{v}_{3}}{\partial \tilde{x}_{1}}\right)^{2}+\left(\frac{\partial \tilde{v}_{2}}{\partial \tilde{x}_{3}}+\epsilon^{2} \frac{\partial \tilde{v}_{3}}{\partial \tilde{x}_{2}}\right)^{2} \\
& +\epsilon^{2}\left(\frac{\partial \tilde{v}_{1}}{\partial \tilde{x}_{2}}+\frac{\partial \tilde{v}_{2}}{\partial \tilde{x}_{1}}\right)^{2}+4 \epsilon^{2}\left(\frac{\partial \tilde{v}_{1}}{\partial \tilde{x}_{1}}\right)^{2} \\
& +4 \epsilon^{2}\left(\frac{\partial \tilde{v}_{2}}{\partial \tilde{x}_{2}}\right)^{2}+4 \epsilon^{2}\left(\frac{\partial \tilde{v}_{1}}{\partial \tilde{x}_{1}}\right)\left(\frac{\partial \tilde{v}_{2}}{\partial \tilde{x}_{2}}\right) .
\end{aligned}
$$

Inversely, the strain-rate tensor may be expressed in terms of the stress tensor as

$$
\dot{\varepsilon}_{i j}=A \sigma_{I I}^{n-1} \sigma_{i j},
$$

where

$$
\sigma_{\|}=\sqrt{\frac{\boldsymbol{\sigma}_{k l} \boldsymbol{\sigma}_{k l}}{2}},
$$

which, in the scaled quantities, reads as

$$
\begin{aligned}
\frac{\partial \tilde{v}_{1}}{\partial \tilde{x}_{1}} & =\epsilon^{-2} C \mathcal{S} \tilde{\boldsymbol{\sigma}}_{11} \\
\frac{\partial \tilde{v}_{2}}{\partial \tilde{x}_{2}} & =\epsilon^{-2} C \mathcal{S} \tilde{\boldsymbol{\sigma}}_{22} \\
\frac{\partial \tilde{v}_{1}}{\partial \tilde{x}_{2}}+\frac{\partial \tilde{v}_{2}}{\partial \tilde{x}_{1}} & =2 \epsilon^{-2} C \mathcal{S} \tilde{\boldsymbol{\sigma}}_{12} \\
\frac{\partial \tilde{v}_{1}}{\partial \tilde{x}_{3}}+\epsilon^{2} \frac{\partial \tilde{v}_{3}}{\partial \tilde{x}_{1}} & =2 \epsilon^{-1} C \mathcal{S} \tilde{\boldsymbol{\sigma}}_{13} \\
\frac{\partial \tilde{v}_{2}}{\partial \tilde{x}_{3}}+\epsilon^{2} \frac{\partial \tilde{v}_{3}}{\partial \tilde{x}_{2}} & =2 \epsilon^{-1} C \mathcal{S} \tilde{\boldsymbol{\sigma}}_{23} \\
\mathcal{S} & =\tilde{\boldsymbol{\sigma}}_{11}^{2}+\tilde{\boldsymbol{\sigma}}_{22}^{2}+\tilde{\boldsymbol{\sigma}}_{11} \tilde{\boldsymbol{\sigma}}_{22}+\tilde{\boldsymbol{\sigma}}_{12}^{2}+\tilde{\boldsymbol{\sigma}}_{13}^{2}+\tilde{\boldsymbol{\sigma}}_{23}^{2} .
\end{aligned}
$$

\section{THE SIA-I ALGORITHM}

In this section, we derive an iterative algorithm for updating velocity and stress fields based on the application of the Banach fixed-point theorem (e.g. Granas, 2003) for the ice-flow problem. The iterations start with the SIA-derived stress and velocity fields, which are then updated by solving an approximate problem that has more convenient numerical properties than the original setting. A crucial issue, convergence of the iterative algorithm, is ensured if the contractivity of the iterations holds. A detailed theoretical analysis of this question is not the subject of this paper, but the numerical examples presented in the following indicate that the algorithm converges for a wide range of icemodel parameters if the projection parameters controlling the iterations are chosen to be sufficiently small.

To derive the algorithm, let us consider the system of Equations (11-13) and assume there is an approximate solution in the $k$ th iterative step, i.e. the field

$$
\vec{u}^{k} \equiv\left(\tilde{p}^{k}, \tilde{\boldsymbol{\sigma}}_{11}^{k}, \tilde{\boldsymbol{\sigma}}_{12}^{k}, \tilde{\boldsymbol{\sigma}}_{22}^{k}, \tilde{\boldsymbol{\sigma}}_{13}^{k}, \tilde{\boldsymbol{\sigma}}_{23}^{k}\right) \text {. }
$$

The solution in the $(k+1)$ th iteration is constructed in a twostep procedure. In the first half-step, we find $\vec{u}^{k+\frac{1}{2}}$ as follows. 
Denoting the exact solution of Equations (11-13) by $\vec{u}$ and defining the increment $\delta \vec{u}^{k+\frac{1}{2}}$ as

$$
\delta \vec{u}^{k+\frac{1}{2}}=\vec{u}-\vec{u}^{k},
$$

the system of Equations (11-13) for $\vec{u}$ may be rewritten as a system of equations for the increment $\delta u^{k+\frac{1}{2}}$, assuming that $\vec{u}^{k}$ is known. We therefore obtain

$$
\begin{aligned}
& -\frac{\partial \delta \tilde{p}^{k+\frac{1}{2}}}{\partial \tilde{x}_{1}} \epsilon+\frac{\partial \delta \tilde{\boldsymbol{\sigma}}_{11}^{k+\frac{1}{2}}}{\partial \tilde{x}_{1}} \epsilon+\frac{\partial \delta \tilde{\boldsymbol{\sigma}}_{12}^{k+\frac{1}{2}}}{\partial \tilde{x}_{2}} \epsilon+\frac{\partial \delta \tilde{\boldsymbol{\sigma}}_{13}^{k+\frac{1}{2}}}{\partial \tilde{x}_{3}} \\
& =\frac{\partial \tilde{p}^{k}}{\partial \tilde{x}_{1}} \epsilon-\frac{\partial \tilde{\boldsymbol{\sigma}}_{11}^{k}}{\partial \tilde{x}_{1}} \epsilon-\frac{\partial \tilde{\boldsymbol{\sigma}}_{12}^{k}}{\partial \tilde{x}_{2}} \epsilon-\frac{\partial \tilde{\boldsymbol{\sigma}}_{13}^{k}}{\partial \tilde{x}_{3}}, \\
& -\frac{\partial \delta \tilde{p}^{k+\frac{1}{2}}}{\partial \tilde{x}_{2}} \epsilon+\frac{\partial \delta \tilde{\boldsymbol{\sigma}}_{12}^{k+\frac{1}{2}}}{\partial \tilde{x}_{1}} \epsilon+\frac{\partial \delta \tilde{\boldsymbol{\sigma}}_{22}^{k+\frac{1}{2}}}{\partial \tilde{x}_{2}} \epsilon+\frac{\partial \delta \tilde{\boldsymbol{\sigma}}_{23}^{k+\frac{1}{2}}}{\partial \tilde{x}_{3}} \\
& =\frac{\partial \tilde{p}^{k}}{\partial \tilde{x}_{2}} \epsilon-\frac{\partial \tilde{\boldsymbol{\sigma}}_{12}^{k}}{\partial \tilde{x}_{1}} \epsilon-\frac{\partial \tilde{\boldsymbol{\sigma}}_{22}^{k}}{\partial \tilde{x}_{2}} \epsilon-\frac{\partial \tilde{\boldsymbol{\sigma}}_{23}^{k}}{\partial \tilde{x}_{3}}, \\
& \frac{\partial \delta \tilde{p}^{k+\frac{1}{2}}}{\partial \tilde{x}_{3}} \epsilon-\frac{\partial \delta \tilde{\boldsymbol{\sigma}}_{13}^{k+\frac{1}{2}}}{\partial \tilde{x}_{1}} \epsilon-\frac{\partial \delta \tilde{\boldsymbol{\sigma}}_{23}^{k+\frac{1}{2}}}{\partial \tilde{x}_{2}} \epsilon+\frac{\partial\left(\delta \tilde{\boldsymbol{\sigma}}_{11}^{k+\frac{1}{2}}+\delta \tilde{\boldsymbol{\sigma}}_{22}^{k+\frac{1}{2}}\right)}{\partial \tilde{x}_{3}} \\
& =-1-\frac{\partial \tilde{p}^{k}}{\partial \tilde{x}_{3}} \epsilon+\frac{\partial \tilde{\boldsymbol{\sigma}}_{13}^{k}}{\partial \tilde{x}_{1}} \epsilon+\frac{\partial \tilde{\boldsymbol{\sigma}}_{23}^{k}}{\partial \tilde{x}_{2}} \epsilon \\
& -\frac{\partial\left(\tilde{\boldsymbol{\sigma}}_{11}^{k}+\tilde{\boldsymbol{\sigma}}_{22}^{k}\right)}{\partial \tilde{x}_{3}} \text {. }
\end{aligned}
$$

We now retain only the stresses $\delta \tilde{p}^{k+\frac{1}{2}}, \delta \tilde{\boldsymbol{\sigma}}_{13}^{k+\frac{1}{2}}$ and $\delta \tilde{\boldsymbol{\sigma}}_{23}^{k+\frac{1}{2}}$ in Equations (42) and (43) and the stress $\delta \tilde{p}^{k+\frac{1}{2}}$ in Equation (44), and neglect all other terms on the lefthand sides of the equations. This approximation exactly corresponds to the traditional SIA approach, assuming that only the retaining stress components are dominant. Here, this approach is applied to the stress increments, $\delta \vec{u}$, only, instead of the complete stress field as in the SIA (e.g. Greve, 1997). Hence, none of the stress components from the previous $k$ th iterative step are omitted on the righthand sides of Equations (42-44). The SIA-like approximation results in the equations for the stress increments as follows

$$
\begin{aligned}
-\frac{\partial \delta \tilde{p}^{k+\frac{1}{2}}}{\partial \tilde{x}_{1}} \epsilon+\frac{\partial \delta \tilde{\boldsymbol{\sigma}}_{13}^{k+\frac{1}{2}}}{\partial \tilde{x}_{3}}= & \frac{\partial \tilde{p}^{k}}{\partial \tilde{x}_{1}} \epsilon-\frac{\partial \tilde{\boldsymbol{\sigma}}_{11}^{k}}{\partial \tilde{x}_{1}} \epsilon \\
& -\frac{\partial \tilde{\boldsymbol{\sigma}}_{12}^{k}}{\partial \tilde{x}_{2}} \epsilon-\frac{\partial \tilde{\boldsymbol{\sigma}}_{13}^{k}}{\partial \tilde{x}_{3}} \\
-\frac{\partial \delta \tilde{p}^{k+\frac{1}{2}}}{\partial \tilde{x}_{2}} \epsilon+\frac{\partial \delta \tilde{\boldsymbol{\sigma}}_{23}^{k+\frac{1}{2}}}{\partial \tilde{x}_{3}}= & \frac{\partial \tilde{p}^{k}}{\partial \tilde{x}_{2}} \epsilon-\frac{\partial \tilde{\boldsymbol{\sigma}}_{12}^{k}}{\partial \tilde{x}_{1}} \epsilon \\
& -\frac{\partial \tilde{\boldsymbol{\sigma}}_{22}^{k}}{\partial \tilde{x}_{2}} \epsilon-\frac{\partial \tilde{\boldsymbol{\sigma}}_{23}^{k}}{\partial \tilde{x}_{3}} \\
\frac{\partial \delta \tilde{p}^{k+\frac{1}{2}}}{\partial \tilde{x}_{3}}= & -1-\frac{\partial \tilde{p}^{k}}{\partial \tilde{x}_{3}}+\frac{\partial \tilde{\boldsymbol{\sigma}}_{13}^{k}}{\partial \tilde{x}_{1}} \epsilon \\
& +\frac{\partial \tilde{\boldsymbol{\sigma}}_{23}^{k}}{\partial \tilde{x}_{2}} \epsilon-\frac{\partial\left(\tilde{\boldsymbol{\sigma}}_{11}^{k}-\tilde{\boldsymbol{\sigma}}_{22}^{k}\right)}{\partial \tilde{x}_{3}}
\end{aligned}
$$

Equation (47) is now integrated along the vertical coordinate $\tilde{x}_{3}$, from the computation point $\tilde{x}_{3}$ to the boundary point $\tilde{f}_{\mathrm{s}}$ which yields the pressure increment $\delta \tilde{p}^{k+\frac{1}{2}}$ at the computation point $\tilde{x}_{3}$. This result is then substituted into Equations (45) and (46) which, after integration along the vertical coordinate, $\tilde{\boldsymbol{x}}_{3}$, gives the increments $\delta \tilde{\boldsymbol{\sigma}}_{13}^{k+\frac{1}{2}}, \delta \tilde{\boldsymbol{\sigma}}_{23}^{k+\frac{1}{2}}$ at the computation point $\tilde{X}_{3}$. The values of the integrands at $\tilde{f}_{\mathrm{s}}$ are determined from the boundary conditions, Equations (16-18). To find them, the same procedure as before is applied. The exact solution is decomposed into the $k$ th iterative-step solution, $\vec{u}^{k}$, and the increment $\delta \vec{u}^{k+\frac{1}{2}}$, and only increments in $\tilde{p}, \tilde{\boldsymbol{\sigma}}_{13}$ and $\tilde{\boldsymbol{\sigma}}_{23}$ are retained to compensate for the discrepancy in adjusting the boundary conditions in the $k$ th iterative step. After some algebraic manipulation, we obtain the boundary conditions for the increments in the form

$$
\begin{aligned}
\delta \tilde{p}^{k+\frac{1}{2}}= & -\tilde{p}^{k}-\tilde{\boldsymbol{\sigma}}_{11}^{k}-\tilde{\boldsymbol{\sigma}}_{22}^{k}+\epsilon^{2}, \\
\delta \tilde{\boldsymbol{\sigma}}_{13}^{k+\frac{1}{2}}= & -\tilde{\boldsymbol{\sigma}}_{13}^{k}+2 \tilde{\boldsymbol{\sigma}}_{11}^{k}\left(\frac{\partial \tilde{f}_{\mathrm{s}}}{\partial \tilde{x}_{1}}\right) \epsilon+\tilde{\boldsymbol{\sigma}}_{12}^{k}\left(\frac{\partial \tilde{f}_{\mathrm{s}}}{\partial \tilde{x}_{2}}\right) \epsilon \\
& +\tilde{\boldsymbol{\sigma}}_{22}^{k}\left(\frac{\partial \tilde{f}_{\mathrm{s}}}{\partial \tilde{x}_{1}}\right) \epsilon+\epsilon^{2}, \\
\delta \tilde{\boldsymbol{\sigma}}_{23}^{k+\frac{1}{2}}= & -\tilde{\boldsymbol{\sigma}}_{23}^{k}+\tilde{\boldsymbol{\sigma}}_{11}^{k}\left(\frac{\partial \tilde{f}_{\mathrm{s}}}{\partial \tilde{x}_{2}}\right) \epsilon+\tilde{\boldsymbol{\sigma}}_{12}^{k}\left(\frac{\partial \tilde{f}_{\mathrm{s}}}{\partial \tilde{x}_{1}}\right) \epsilon \\
& +2 \tilde{\boldsymbol{\sigma}}_{22}^{k}\left(\frac{\partial \tilde{f}_{\mathrm{s}}}{\partial \tilde{x}_{2}}\right) \epsilon+\epsilon^{2},
\end{aligned}
$$

that are evaluated at $\tilde{x}_{3}=\tilde{f}_{\mathrm{s}}\left(\tilde{x}_{1}, \tilde{x}_{2}\right)$ and we do not explicitly write the terms with $\epsilon^{2}$, because they will be excluded from the computations in the algorithm. Strictly speaking, this cannot be justified by the introduced scaling because we do not assume the scaled quantities and their spatial derivatives to be of order unity. What we present may hence be viewed as merely a formal procedure, which will be justified only by the final performance of the algorithm.

To sum up, the integration of Equation (47), followed by the integration of Equations (45) and (46), with the use of Equations (48-50), now results in the following formulae for the stress increments $\delta \tilde{p}^{k+\frac{1}{2}}, \delta \tilde{\boldsymbol{\sigma}}_{13}^{k+\frac{1}{2}}$ and $\delta \tilde{\boldsymbol{\sigma}}_{23}^{k+\frac{1}{2}}$ :

$$
\begin{aligned}
\delta \tilde{p}^{k+\frac{1}{2}}\left(\cdot, \tilde{x}_{3}\right)= & -\tilde{p}^{k}\left(\cdot, \tilde{x}_{3}\right)-\tilde{\boldsymbol{\sigma}}_{11}^{k}\left(\cdot, \tilde{x}_{3}\right)-\tilde{\boldsymbol{\sigma}}_{22}^{k}\left(\cdot, \tilde{x}_{3}\right) \\
& +\left(\tilde{f}_{\mathrm{s}}(\cdot)-\tilde{x}_{3}\right) \\
& -\epsilon \frac{\partial}{\partial \tilde{x}_{1}} \int_{\tilde{x}_{3}}^{\tilde{f}_{s}(\cdot)} \tilde{\boldsymbol{\sigma}}_{13}^{k}\left(\cdot, \tilde{x}_{3}^{\prime}\right) \mathrm{d} \tilde{x}_{3}^{\prime} \\
& -\epsilon \frac{\partial}{\partial \tilde{x}_{2}} \int_{\tilde{x}_{3}}^{\tilde{f}_{s}(\cdot)} \tilde{\boldsymbol{\sigma}}_{23}^{k}\left(\cdot, \tilde{x}_{3}^{\prime}\right) \mathrm{d} \tilde{x}_{3}^{\prime} \\
& +\epsilon \tilde{\boldsymbol{\sigma}}_{13}^{k}\left(\cdot, \tilde{f}_{\mathrm{s}}(\cdot)\right) \frac{\partial \tilde{f}_{\mathrm{s}}(\cdot)}{\partial \tilde{x}_{1}} \\
& +\epsilon \tilde{\boldsymbol{\sigma}}_{23}^{k}\left(\cdot, \tilde{f}_{\mathrm{s}}(\cdot)\right) \frac{\partial \tilde{f}_{\mathrm{s}}(\cdot)}{\partial \tilde{x}_{2}}+\epsilon^{2}
\end{aligned}
$$

$$
\begin{aligned}
\delta \tilde{\boldsymbol{\sigma}}_{13}^{k+\frac{1}{2}}\left(\cdot, \tilde{x}_{3}\right)= & -\tilde{\boldsymbol{\sigma}}_{13}^{k}\left(\cdot, \tilde{x}_{3}\right)-\epsilon \frac{\partial \tilde{f}_{\mathrm{s}}(\cdot)}{\partial \tilde{x}_{1}}\left(\tilde{f}_{\mathrm{s}}(\cdot)-\tilde{x}_{3}\right) \\
& +2 \epsilon \frac{\partial}{\partial \tilde{x}_{1}} \int_{\tilde{x}_{3}}^{\tilde{f}_{s}(\cdot)} \tilde{\boldsymbol{\sigma}}_{11}^{k}\left(\cdot, \tilde{x}_{3}^{\prime}\right) \mathrm{d} \tilde{x}_{3}^{\prime} \\
& +\epsilon \frac{\partial}{\partial \tilde{x}_{2}} \int_{\tilde{x}_{3}}^{\tilde{f}_{s}(\cdot)} \tilde{\boldsymbol{\sigma}}_{12}^{k}\left(\cdot, \tilde{x}_{3}^{\prime}\right) \mathrm{d} \tilde{x}_{3}^{\prime} \\
& +\epsilon \frac{\partial}{\partial \tilde{x}_{1}} \int_{\tilde{x}_{3}}^{\tilde{f}_{s}(\cdot)} \tilde{\boldsymbol{\sigma}}_{22}^{k}\left(\cdot, \tilde{x}_{3}^{\prime}\right) \mathrm{d} \tilde{x}_{3}^{\prime}+\epsilon^{2},
\end{aligned}
$$




$$
\begin{aligned}
\delta \tilde{\boldsymbol{\sigma}}_{23}^{k+\frac{1}{2}}\left(\cdot, \tilde{x}_{3}\right)= & -\tilde{\boldsymbol{\sigma}}_{23}^{k}\left(\cdot, \tilde{x}_{3}\right)-\epsilon \frac{\partial \tilde{f}_{s}(\cdot)}{\partial \tilde{x}_{2}}\left(\tilde{f}_{\mathrm{s}}(\cdot)-\tilde{x}_{3}\right) \\
& +\epsilon \frac{\partial}{\partial \tilde{x}_{2}} \int_{\tilde{x}_{3}}^{\tilde{f}_{s}(\cdot)} \tilde{\boldsymbol{\sigma}}_{11}^{k}\left(\cdot, \tilde{x}_{3}^{\prime}\right) \mathrm{d} \tilde{x}_{3}^{\prime} \\
& +\epsilon \frac{\partial}{\partial \tilde{x}_{1}} \int_{\tilde{x}_{3}}^{\tilde{f}_{s}(\cdot)} \tilde{\boldsymbol{\sigma}}_{12}^{k}\left(\cdot, \tilde{x}_{3}^{\prime}\right) \mathrm{d} \tilde{x}_{3}^{\prime} \\
& +2 \epsilon \frac{\partial}{\partial \tilde{x}_{2}} \int_{\tilde{x}_{3}}^{\tilde{f}_{s}(\cdot)} \tilde{\boldsymbol{\sigma}}_{22}^{k}\left(\cdot, \tilde{x}_{3}^{\prime}\right) \mathrm{d} \tilde{x}_{3}^{\prime}+\epsilon^{2},
\end{aligned}
$$

where the dot in $(\cdot)$ stands for the pair $\left(\tilde{x}_{1}, \tilde{x}_{2}\right)$ for brevity and again terms with $\epsilon^{2}$ are not considered further and therefore not explicitly written.

We now define the updated solution in the $\left(k+\frac{1}{2}\right)$ step as

$$
\vec{u}^{k+\frac{1}{2}}=\vec{u}^{k}+\theta_{1} \delta \vec{u}^{k+\frac{1}{2}},
$$

where $\theta_{1} \in(0,1)$ is the first projection parameter of the iterative scheme. Note that, by the previous derivations, we consider

$$
\delta \vec{u}^{k+\frac{1}{2}}=\left(\delta \tilde{p}^{k+\frac{1}{2}}, 0,0,0, \delta \tilde{\boldsymbol{\sigma}}_{13}^{k+\frac{1}{2}}, \delta \tilde{\boldsymbol{\sigma}}_{23}^{k+\frac{1}{2}}\right),
$$

that is, only these three stress components are updated in the first half-step.

In the second half-step, the consistency of the stress field with the velocity field must be ensured, that is, the rheological equation must be adjusted.

First, the terms with $\epsilon^{2}$ on the lefthand sides of Equations (37) and (38) are neglected and the result is integrated along the vertical coordinate $\tilde{x}_{3}$ from the glacier bed $\tilde{f}_{\mathrm{b}}$ to the computation point $\tilde{x}_{3}$. Making use of the no-slip boundary condition, Equation (20), and considering the updated stress field $\boldsymbol{\sigma}_{i j}^{k+\frac{1}{2}}$, we obtain

$$
\begin{aligned}
& \tilde{v}_{1}^{k+\frac{1}{2}}\left(\cdot, \tilde{x}_{3}\right)=2 \epsilon^{-1} \mathrm{C} \int_{\tilde{f}_{\mathrm{b}}(\cdot)}^{\tilde{x}_{3}} S^{k+\frac{1}{2}}\left(\cdot, \tilde{x}_{3}^{\prime}\right) \tilde{\boldsymbol{\sigma}}_{13}^{k+\frac{1}{2}}\left(\cdot, \tilde{x}_{3}^{\prime}\right) \mathrm{d} \tilde{x}_{3}^{\prime}, \\
& \tilde{v}_{2}^{k+\frac{1}{2}}\left(\cdot, \tilde{x}_{3}\right)=2 \epsilon^{-1} \mathrm{C} \int_{\tilde{f}_{\mathrm{b}}(\cdot)}^{\tilde{x}_{3}} S^{k+\frac{1}{2}}\left(\cdot, \tilde{x}_{3}^{\prime}\right) \tilde{\boldsymbol{\sigma}}_{23}^{k+\frac{1}{2}}\left(\cdot, \tilde{x}_{3}^{\prime}\right) \mathrm{d} \tilde{x}_{3}^{\prime} .
\end{aligned}
$$

The velocity, $\tilde{v}_{3}^{k+\frac{1}{2}}$, is then obtained by integration of the incompressibility condition, Equation (14), from $\tilde{f}_{\mathrm{b}}$ to $\tilde{x}_{3}$. Making use of the no-slip boundary condition, Equation (20), we obtain

$$
\tilde{v}_{3}^{k+\frac{1}{2}}\left(\cdot, \tilde{x}_{3}\right)=-\int_{\tilde{f}_{\mathrm{b}}(\cdot)}^{\tilde{x}_{3}}\left(\frac{\partial \tilde{v}_{1}^{k+\frac{1}{2}}}{\partial \tilde{x}_{1}}+\frac{\partial \tilde{v}_{2}^{k+\frac{1}{2}}}{\partial \tilde{x}_{2}}\right)\left(\cdot, \tilde{x}_{3}^{\prime}\right) \mathrm{d} \tilde{x}_{3}^{\prime} .
$$

This completes the determination of the velocity field $\vec{v}^{k+\frac{1}{2}}$.

This field is now used to update the stress components from the rheological equation, that consequently reduces the inconsistency of the updated velocity field with stresses. The substitution of the velocity, $\vec{v}^{k+\frac{1}{2}}$, into the rheological equations (24-31) yields the stress components that form a stress vector denoted by $\vec{u}^{\star k+\frac{1}{2}}$. The new $\vec{u}^{k+1}$ is finally defined as a convex combination of the previous solution given by Equation (54) and the rheologically consistent solution $\vec{u}^{\star k+\frac{1}{2}}$, i.e. we define

$$
\vec{u}^{k+1}=\vec{u}^{k+\frac{1}{2}}\left(1-\theta_{2}\right)+\theta_{2} \vec{u}^{\star n+\frac{1}{2}},
$$

Table 1. Values of physical parameters

\begin{tabular}{llcc}
\hline Symbol & Constant & Value & Unit \\
\hline A & Ice-flow parameter & $10^{-16}$ & $\mathrm{~Pa} \mathrm{a}^{-1}$ \\
$\rho$ & Ice density & 910 & $\mathrm{~kg} \mathrm{~m}^{-3}$ \\
$g$ & Gravitational acceleration & 9.81 & $\mathrm{~m} \mathrm{~s}^{-2}$ \\
\hline
\end{tabular}

where $\theta_{2} \in(0,1)$ is the second projection parameter of the iterative scheme.

We call the presented approach that iteratively improves the SIA solution the SIA-I algorithm. Its computational steps may symbolically be depicted by the following scheme, starting from $u^{k}$ :

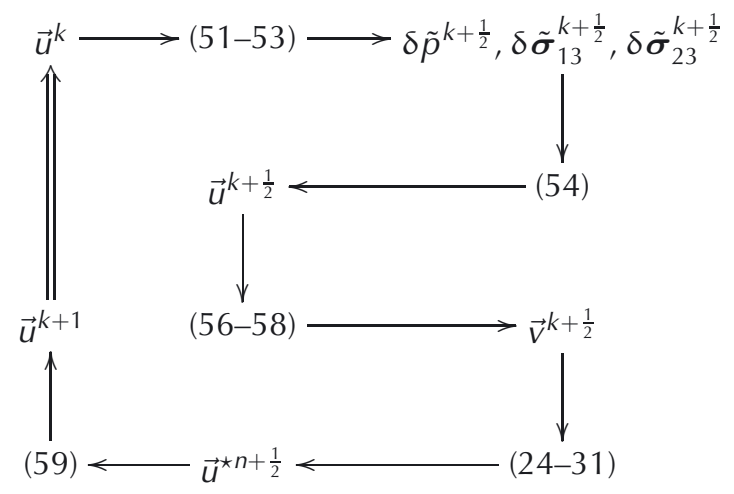

\section{NUMERICAL SIMULATION}

In this section, we present numerical results obtained by the SIA-I approach for the ISMIP-HOM (Ice Sheet Model Intercomparison Project-Higher-Order ice-sheet Model) experiment (http://homepages.ulb.ac.be/ fpattyn/ismip/).

Our approach has been incorporated into experiments $A$ and B (model oso1) (see Pattyn and others, 2008, for a discussion of results and model outputs). Here, we present the results for experiment $A$ where the no-slip condition is considered at the glacier base. We also discuss the performance of the SIA-I algorithm for experiment C, where basal sliding with a prescribed sliding law is considered.

\section{ISMIP-HOM experiment A}

The problem is set up as follows. The experiment involves a Stokes flow problem, no slip at the bed, stress-free conditions at the surface and the ice is considered isothermal. The values of the physical parameters used are given in Table 1.

The glacier has a square base of size $[L] \times[L]$. The upper and lower surfaces are given (in meters) by

$$
\begin{gathered}
f_{\mathrm{s}}\left(x_{1}, x_{2}\right)=-x_{1} \tan \alpha, \quad \alpha=0.5^{\circ}, \\
f_{\mathrm{b}}\left(x_{1}, x_{2}\right)=f_{\mathrm{s}}\left(x_{1}, x_{2}\right)-1000+500 \sin \left(\omega x_{1}\right) \sin \left(\omega x_{2}\right),
\end{gathered}
$$

with

$$
\omega=\frac{2 \pi}{[L]} .
$$

At the sides, the periodic boundary conditions are prescribed: $\forall x_{1} \in\langle 0, L\rangle, \forall x_{3} \in\left\langle f_{\mathrm{b}}(\cdot), f_{\mathrm{s}}(\cdot)\right\rangle: \vec{v}\left(x_{1}, 0, x_{3}\right)=\vec{v}\left(x_{1},[L], x_{3}\right)$, 

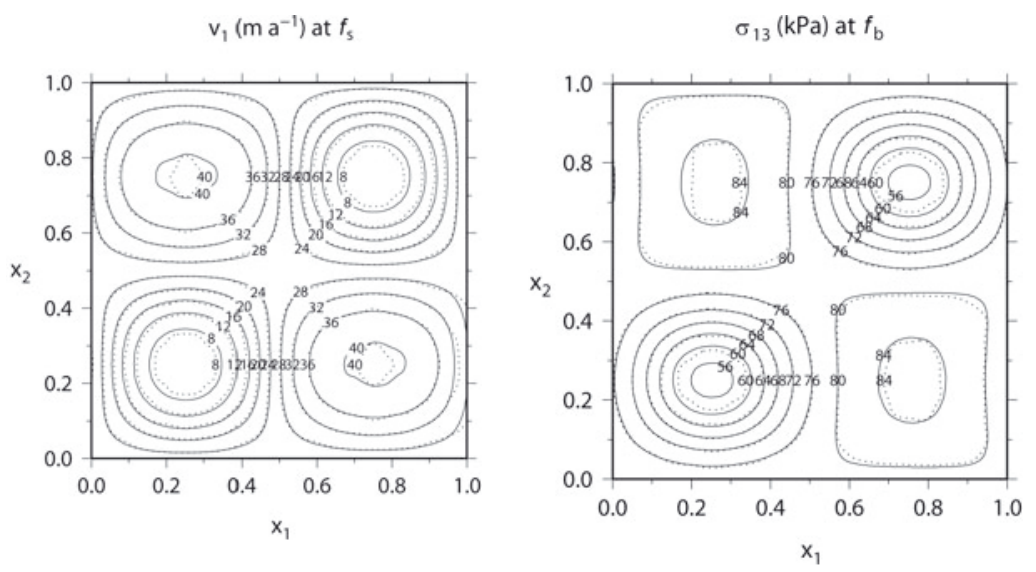

$\mathrm{v}_{2}\left(\mathrm{~m} \mathrm{a}^{-1}\right)$ at $f_{\mathrm{s}}$

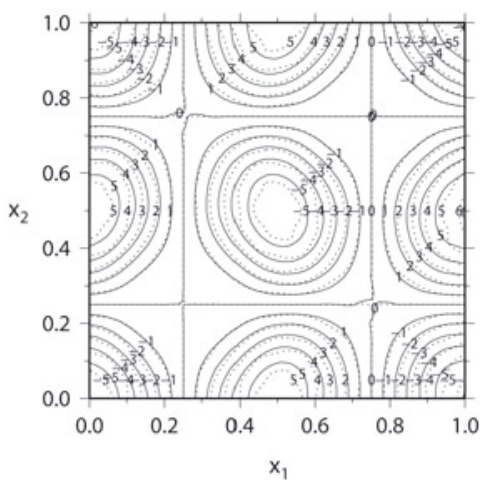

$\sigma_{23}(\mathrm{kPa})$ at $f_{\mathrm{b}}$

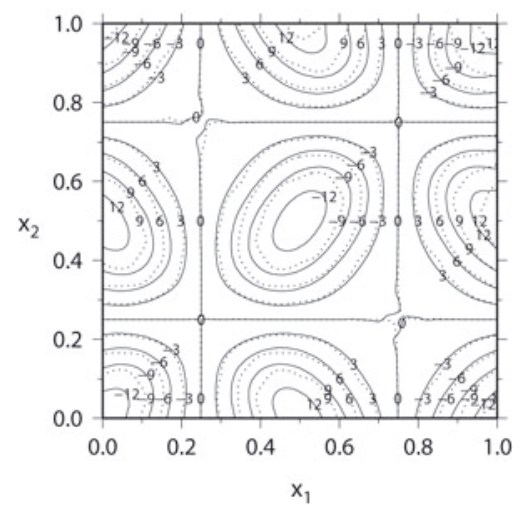

$v_{3}\left(\mathrm{~m} \mathrm{a}^{-1}\right)$ at $f_{\mathrm{s}}$

$\Delta \mathrm{p}(\mathrm{kPa})$ at $f_{\mathrm{b}}$
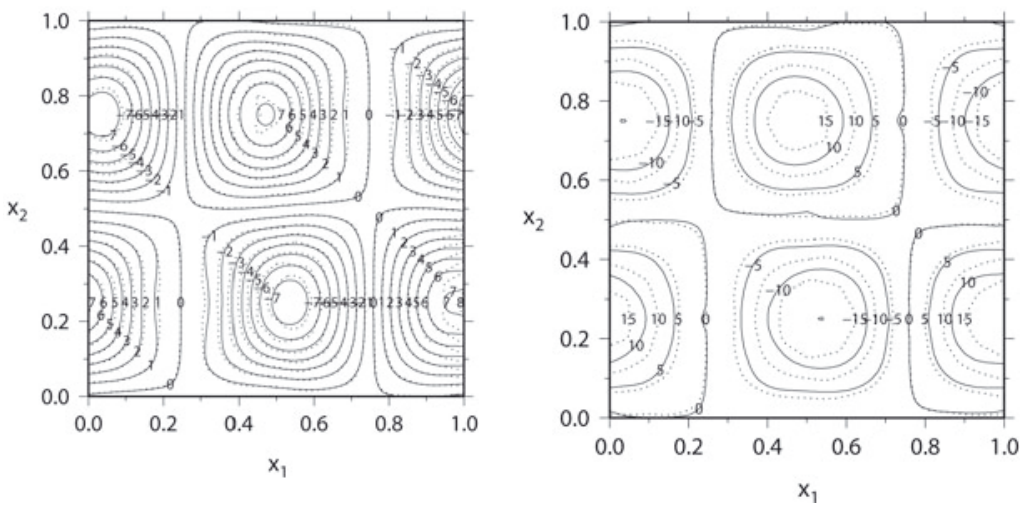

Fig. 1. Comparison of the surface velocity fields $\left(\mathrm{ma}^{-1}\right)$, and stress components, $\boldsymbol{\sigma}_{13}, \boldsymbol{\sigma}_{23}$, and the pressure difference, $\Delta p=p-H_{\rho} g$ at the bottom (kPa), obtained by the SIA-I solver (full line labeled diagonally) and the full-Stokes solver (dotted line labeled horizontally), respectively, for the aspect ratio $\epsilon=\frac{1}{20}$.

$\forall x_{2} \in\langle 0, L\rangle, \forall x_{3} \in\left\langle f_{\mathrm{b}}(\cdot), f_{\mathrm{s}}(\cdot)\right\rangle: \vec{v}\left(0, x_{2}, x_{3}\right)=\vec{v}\left([L], x_{2}, x_{3}\right)$

The plotted quantities are the velocities $v_{1}, v_{2}, v_{3}$ at the upper surface (in $\mathrm{m} \mathrm{a}^{-1}$ ) and stress components $\boldsymbol{\sigma}_{13}, \boldsymbol{\sigma}_{23}, \Delta p=$ $p-H \rho g$ at the bottom (in $\mathrm{kPa}$ ), $H=f_{\mathrm{s}}-f_{\mathrm{b}}$. All quantities are mapped onto the scaled domain $\langle 0,1\rangle \times\langle 0,1\rangle$. The numerical implementation includes the transformation of the problem into stretched coordinates, as usual in glaciology (e.g. Pattyn, 2003). The glacier flow computed by the SIAI approach is checked against a finite-difference full-Stokes solver (see Appendix). For a more detailed comparison with other solvers, see the results of the ISMIP-HOM experiment A (Pattyn and others, 2008).

\section{RESULTS}

\section{Results for $\epsilon=\frac{1}{20}$}

The SIA-I solution is computed with the projection parameters $\theta_{1}=0.2$ and $\theta_{2}=0.05$. The results are stored in a staggered grid of dimensions $41 \times 41 \times 41$, where one type of node contains the velocity components $\tilde{v}_{1}, \tilde{v}_{2}, \tilde{v}_{3}$, while the other nodes contain the stress-tensor components $\tilde{\boldsymbol{\sigma}}_{11}, \tilde{\boldsymbol{\sigma}}_{22}, \tilde{\boldsymbol{\sigma}}_{12}, \tilde{\boldsymbol{\sigma}}_{13}, \tilde{\boldsymbol{\sigma}}_{23}$ and pressure $\tilde{p}$. The SIA-I solution, obtained after 60 iterations, is shown in Figure 1 (full lines). The computation was performed on an Intel Pentium 4, $3.2 \mathrm{GHz}$ computer and took $\sim 52 \mathrm{~s}$. More details on the computational costs of the SIA-I are given below.

The full-Stokes solution (dotted lines in Fig. 1) was obtained by the standard finite-difference method (see 

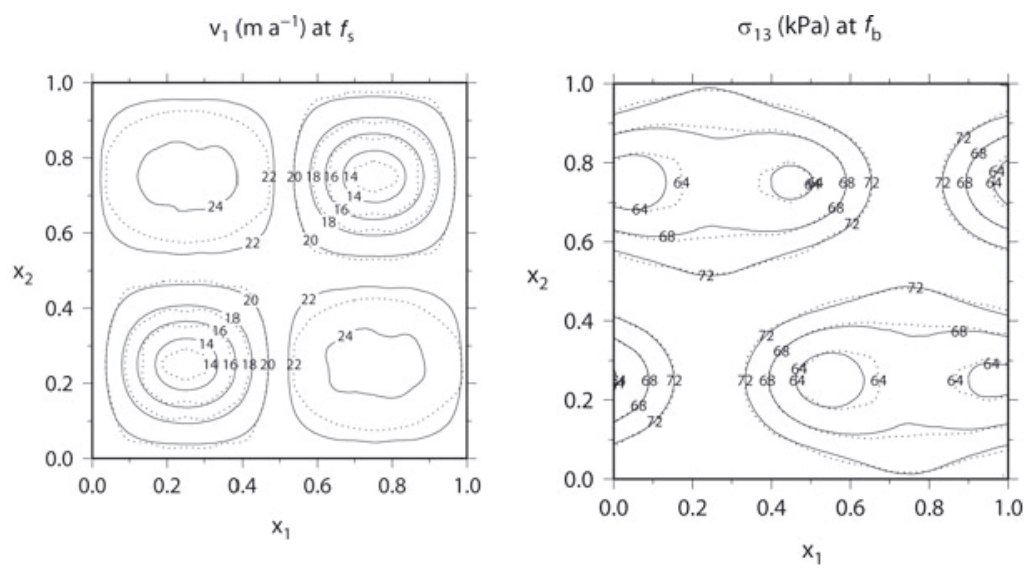

$\mathrm{v}_{2}\left(\mathrm{~m} \mathrm{a}^{-1}\right)$ at $f_{\mathrm{s}}$

$\sigma_{23}(\mathrm{kPa})$ at $f_{\mathrm{b}}$

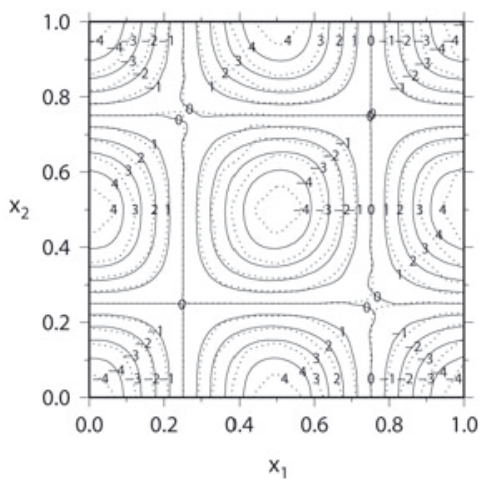

$v_{3}\left(\mathrm{~m} \mathrm{a}^{-1}\right)$ at $f_{\mathrm{s}}$
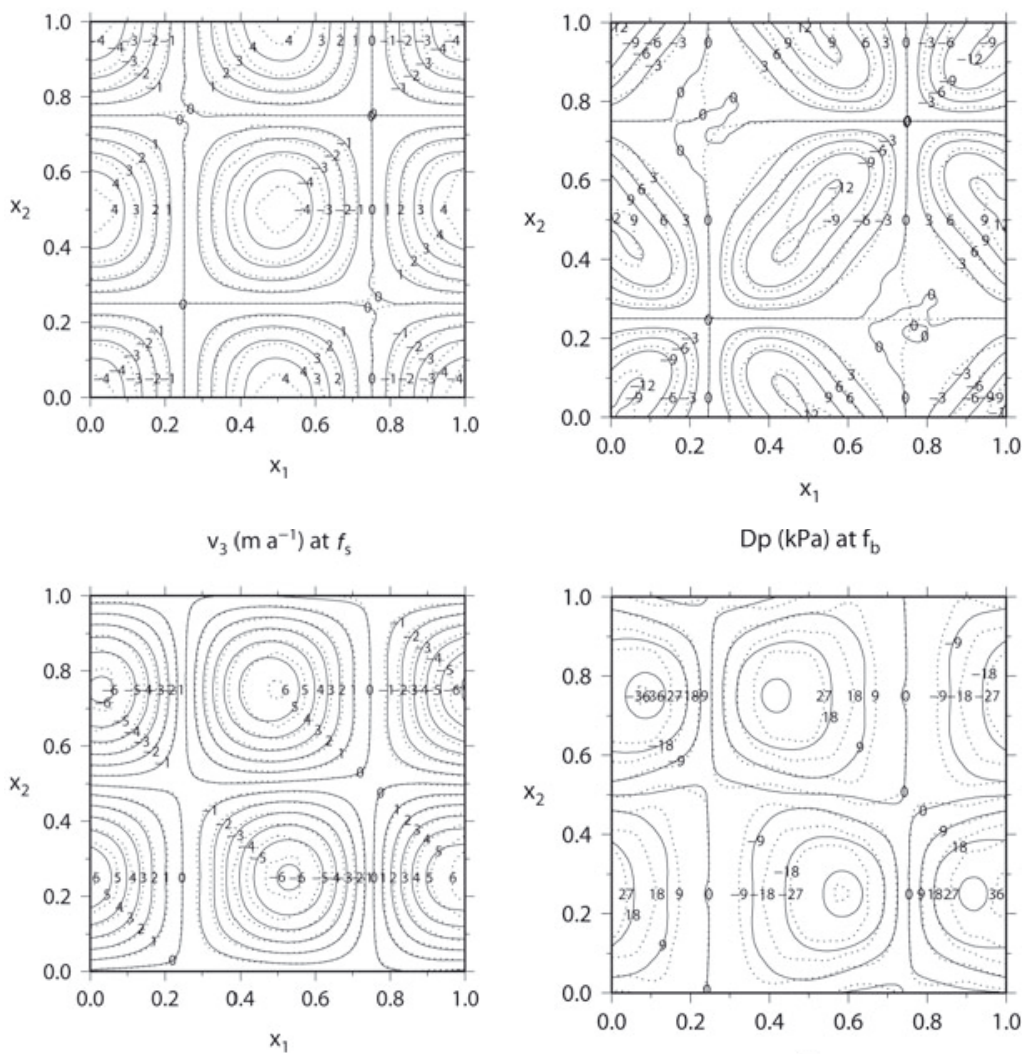

$D p(k P a)$ at $f_{b}$

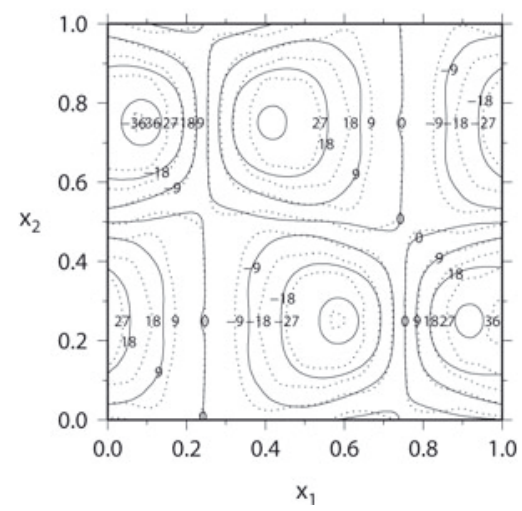

Fig. 2. As for Figure 1 but for $\epsilon=\frac{1}{10}$.

Appendix). It was started from the SIA-I solution on a staggered $20 \times 20 \times 20$ grid. The iterative updating of viscosity was stopped after 40 iterations, when the results were not changing within a specified tolerance. Figure 1 shows an almost perfect agreement between the SIA-I and finite-difference resulting surface velocities and a minor quantitative mismatch for the bottom stress components $\boldsymbol{\sigma}_{13}$ and $\boldsymbol{\sigma}_{23}$. The main difference appears, however, in the bottom-pressure difference, $\Delta p$.

\section{Results for $\epsilon=\frac{1}{\mathbf{1 0}}$}

The SIA-I solution is computed with the projection parameters $\theta_{1}=0.2$ and $\theta_{2}=0.02$. The resolution of the computational domain for both solutions was the same as in the previous case. The SIA-I solution, obtained now after 100 iterations to achieve the required tolerance (Fig. 2, full

lines), is again compared with the full-Stokes solution (dotted lines) which was obtained by the finite-difference approach for 40 iterative updates of viscosity.

Inspecting Figure 2, we see rather good agreement between both the solutions, in particular for the velocities. Again, the largest difference appears in the pressure difference, $\Delta p$. It is noted that the SIA-I solution is smoother than the finite-difference solution, indicating possibly some numerical instabilities in the finite-difference solver.

To estimate the order of improvement of the SIA-I solution compared to the SIA solution, Figure 3 plots the SIA solution for $v_{1}$ and $v_{3}$ at the surface ( $v_{2}$ is identically zero) and $\boldsymbol{\sigma}_{13}$ at the bottom ( $\boldsymbol{\sigma}_{23}$ and $\Delta p$ are identically zero). Comparing Figure 2 with Figure 3, we see that the SIA-I solution differs significantly from the SIA solution, demonstrating that the SIA-I approach is capable of providing a more accurate solution of glacier flow. 


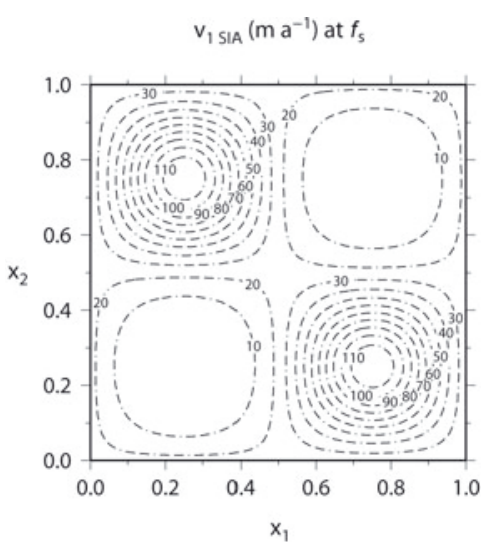

$v_{3 \text { SIA }}\left(\mathrm{m} \mathrm{a}^{-1}\right)$ at $f_{\mathrm{s}}$

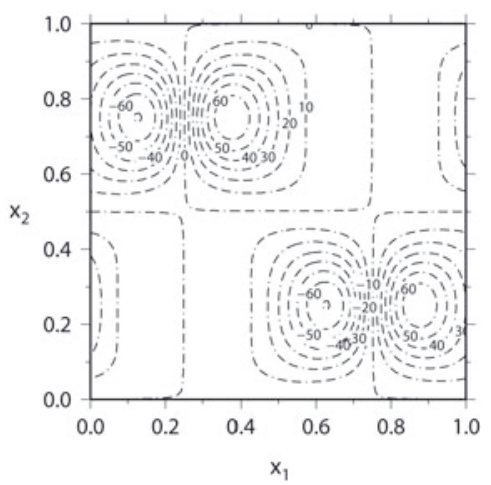

$\sigma_{13 \text { SIA }}(\mathrm{kPa})$ at $f_{\mathrm{b}}$

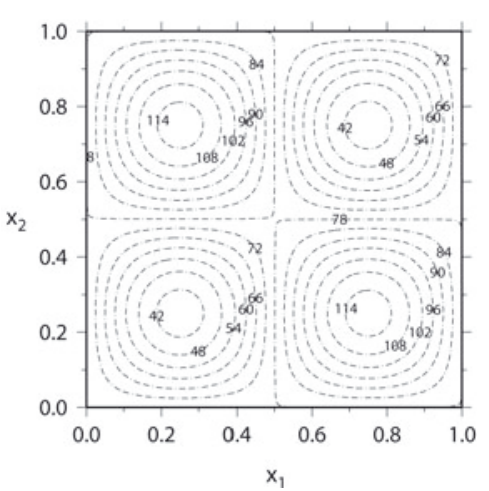

Fig. 3. The SIA solution for the aspect ratio $\epsilon=\frac{1}{10}$. Note that the field quantities not shown here $\left(v_{2}\right.$ at $f_{\mathrm{s}}$ and $\Delta p, \sigma_{23}$, at $\left.f_{\mathrm{b}}\right)$ are identically equal to zero in this case.

As demonstrated in the following section, the convergence of the SIA-I algorithm worsens with increasing aspect ratio, $\epsilon$. This can be overcome, to some extent, by choosing sufficiently small projection parameters $\theta_{1}, \theta_{2}$, but a threshold aspect-ratio value appears to exist for the practical usability of our method. For the current geometry setting from the ISMIP-HOM experiment $A$, this value is $\frac{1}{10}$.

\section{Convergence of the SIA-I algorithm}

In this section, we demonstrate how convergence is affected by varying the aspect ratio, $\epsilon$, and the magnitudes of the projection parameters, $\theta_{1}, \theta_{2}$. We perform all runs with the ISMIP-HOM experiment A set-up.

The convergence rate is inspected by checking the evolution of errors of linear momentum balances, rheology equations and equation of continuity, respectively. These
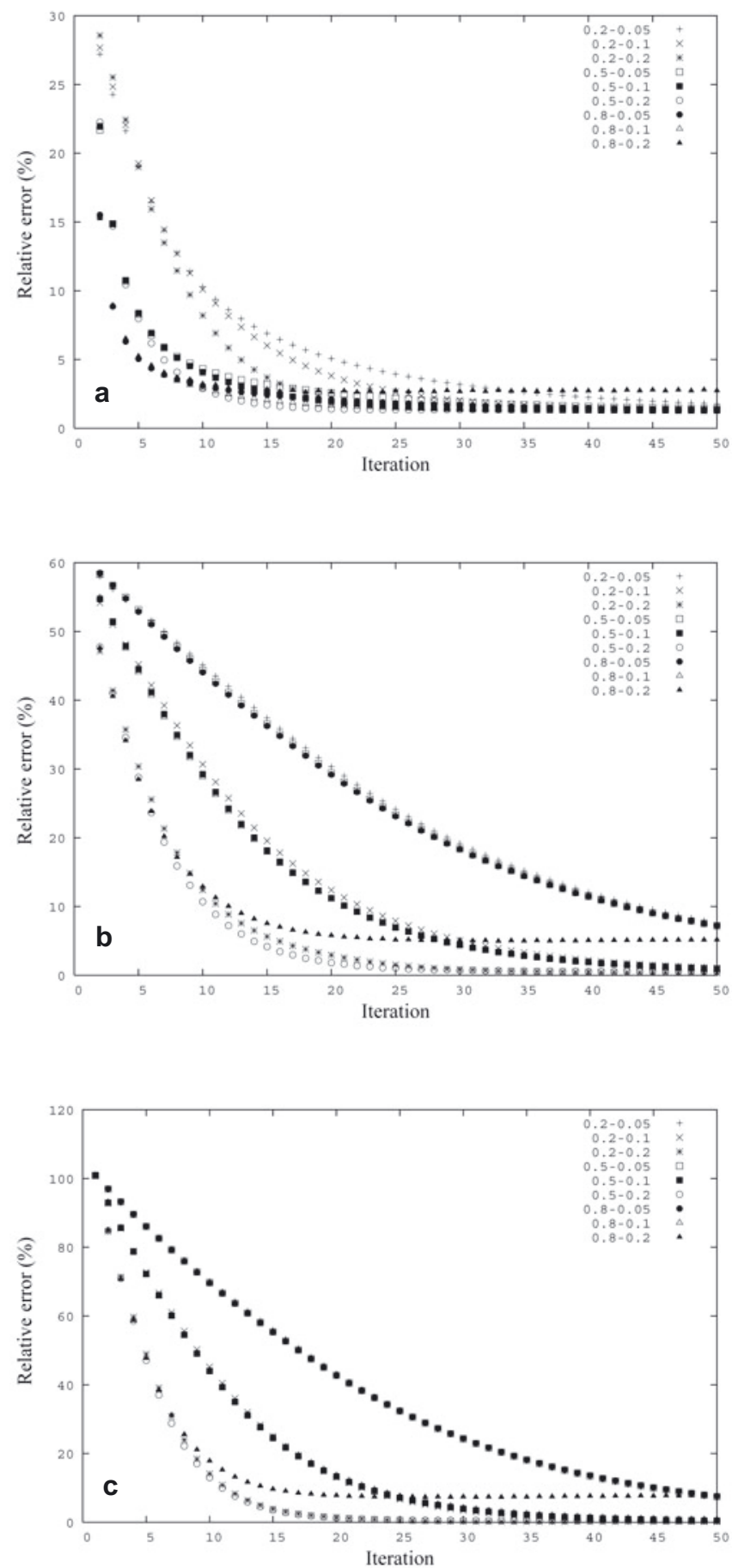

Fig. 4. The evolution of the averaged relative error of (a) linear momentum balances, (b) rheology equations and (c) equation of continuity, for various combinations of the projection parameters $\theta_{1}$ and $\theta_{2}$. The labels read as, for example, '0.2-0.05': $\theta_{1}=0.2$, $\theta_{2}=0.05$. The results apply to the case of a spatial resolution $31 \times 31 \times 31$ and aspect ratio $\frac{1}{80}$.

errors are defined as follows. All the equations are evaluated at the nodes using the discretization of spatial derivatives by two-point symmetric finite differences. If we had an analytical solution, i.e. a solution satisfying the equations exactly in the limit of an infinitesimally small discretization, such a procedure would provide the so-called discretization error. In the case of the SIA-I solution, there is an additional approximation error, resulting from the fact that only an approximation to the full-Stokes problem is solved at each 
SIA-I iteration. We divide the error by the magnitude of the largest term in each particular equation and obtain the relative error at each node. For conciseness we first average these errors over the nodes and then compute one average value from the three linear momentum balance errors, one from the five rheology equations' errors and finally one continuity equation error.

In Figure 4 we plot the total (discretization plus approximation) errors of the SIA-I solution for various combinations of the projection parameters $\theta_{1}=0.2,0.5,0.8, \theta_{2}=$ $0.2,0.1,0.05$, a fixed aspect ratio, $\epsilon=\frac{1}{80}$, and spatial resolution $31 \times 31 \times 31$. For all cases the overall error decreases and eventually reaches a limit (except for the uppermost curves in the middle and bottom panels, where more iterations would be needed to reach the limit). As documented for $\theta_{1}=0.8$ and $\theta_{2}=0.2$ (black triangles), when the projection parameters are chosen to be too large, the solution is scattered by a persistent high-frequency noise preventing the error from dropping below a certain value.

Observe that for, for example, $\theta_{1}=0.5$ and $\theta_{2}=0.2$, the error decreases relatively quickly and a sufficiently accurate solution is obtained after 20 iterations. We also see that below a certain critical value of the projection parameters, the convergence speeds up with their increase, while above the critical threshold the too-large projection parameters induce high-frequency scattering of the output. This may be connected to the spatial resolution since the sequence of successive iterative solutions may formally be viewed as a time-discretized evolution, and as the spatial dependency of field variables is also discretized by finite differences, one may expect a criterion, analogous to the Courant criterion (Press and others, 1992), to be fulfilled to ensure stability of the algorithm. For a given spatial resolution, this criterion would constrain the maximum values of the projection parameters, $\theta_{1}$ and $\theta_{2}$, that control the evolution in 'time'.

Next, in Figure 5, we inspect the role of the aspect ratio, $\epsilon$. Since the derivation of the SIA-I approach requires $\epsilon$ be sufficiently small, there is a threshold value of $\epsilon$ above which the SIA-I algorithm will not converge. Figure 5 plots the errors for aspect ratios $\epsilon=\frac{1}{5}, \frac{1}{10}, \frac{1}{20}, \frac{1}{40}, \frac{1}{80}, \frac{1}{160}$ and a fixed spatial resolution $31 \times 31 \times 31$. The projection parameters are chosen as $\theta_{1}=0.2$ and $\theta_{2}=0.02$, for all computations. Figure 5 clearly demonstrates the key role of the aspect ratio, $\epsilon$, for convergence of the SIA-I algorithm. For the chosen projection parameters, $\theta_{1}, \theta_{2}$, the value $\epsilon=\frac{1}{10}$ is the threshold and for larger aspect ratios the algorithm fails to converge.

In summary, whether the SIA-I algorithm converges and how fast it does so is a matter of several coupled factors. For a sufficiently small aspect ratio, $\epsilon$, (less than $\frac{1}{10}$ for the ISMIP-HOM A experiment), the algorithm converges by choosing projection parameters, $\theta_{1}, \theta_{2}$, below certain threshold values, dependent on both the aspect ratio and the spatial resolution, and the convergence of the algorithm improves by approaching these critical values from below. Moreover, the critical values decrease with increasing aspect ratio, $\epsilon$; as a result, for $\epsilon>\frac{1}{10}$ it is impossible to reach convergence within the ISMIP-HOM A experimental set-up.

\section{Performance of the SIA-I for other than the no-slip boundary condition, ISMIP-HOM experiment C}

The SIA-I algorithm as described above may easily be modified to allow a Dirichlet boundary condition on the velocity at the glacier bed, that is the condition $\vec{v}\left(\cdot, f_{\mathrm{b}}(\cdot)\right)=$ $\vec{v}^{0}(\cdot)$. We merely modify Equations (56-58) as follows:
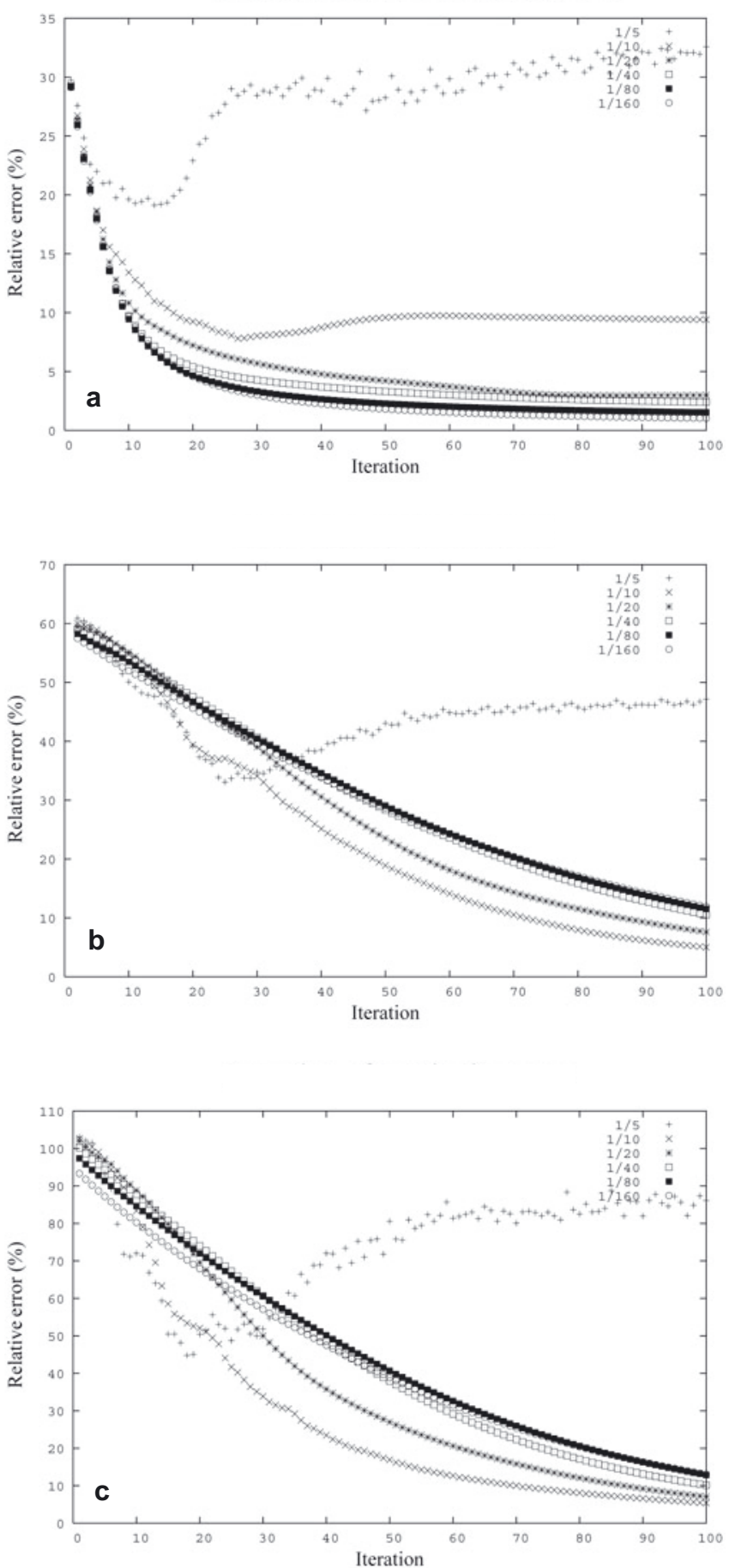

Fig. 5. The evolution of averaged relative error of (a) linear momentum balances, (b) rheology equations and (c) equation of continuity, for various aspect ratios $\epsilon=\frac{1}{5}, \frac{1}{10}, \frac{1}{20}, \frac{1}{40}, \frac{1}{80}, \frac{1}{160}$ and for a fixed spatial resolution $31 \times 31 \times 31$. The results apply to $\theta_{1}=0.2$ and $\theta_{2}=0.02$.

$$
\begin{aligned}
& \tilde{v}_{1}^{k+\frac{1}{2}}\left(\cdot, \tilde{x}_{3}\right) \\
& \quad=\tilde{v}_{1}^{0}(\cdot)+2 \epsilon^{-1} C \int_{\tilde{f}_{\mathrm{b}}(\cdot)}^{\tilde{x}_{3}} S^{k+\frac{1}{2}}\left(\cdot, \tilde{x}_{3}^{\prime}\right) \tilde{\boldsymbol{\sigma}}_{13}^{k+\frac{1}{2}}\left(\cdot, \tilde{x}_{3}^{\prime}\right) \mathrm{d} \tilde{x}_{3}^{\prime}, \\
& \tilde{v}_{2}^{k+\frac{1}{2}}\left(\cdot, \tilde{x}_{3}\right) \\
& \quad=\tilde{v}_{2}^{0}(\cdot)+2 \epsilon^{-1} C \int_{\tilde{f}_{\mathrm{b}}(\cdot)}^{\tilde{x}_{3}} S^{k+\frac{1}{2}}\left(\cdot, \tilde{x}_{3}^{\prime}\right) \tilde{\boldsymbol{\sigma}}_{23}^{k+\frac{1}{2}}\left(\cdot, \tilde{x}_{3}^{\prime}\right) \mathrm{d} \tilde{x}_{3}^{\prime}, \\
& \tilde{v}_{3}^{k+\frac{1}{2}}\left(\cdot, \tilde{x}_{3}\right) \\
& \quad=\tilde{v}_{3}^{0}(\cdot)-\int_{\tilde{f}_{\mathrm{b}}(\cdot)}^{\tilde{x}_{3}}\left(\frac{\partial \tilde{v}_{1}^{k+\frac{1}{2}}}{\partial \tilde{x}_{1}}+\frac{\partial \tilde{v}_{2}^{k+\frac{1}{2}}}{\partial \tilde{x}_{2}}\right)\left(\cdot, \tilde{x}_{3}^{\prime}\right) \mathrm{d} \tilde{x}_{3}^{\prime} .
\end{aligned}
$$



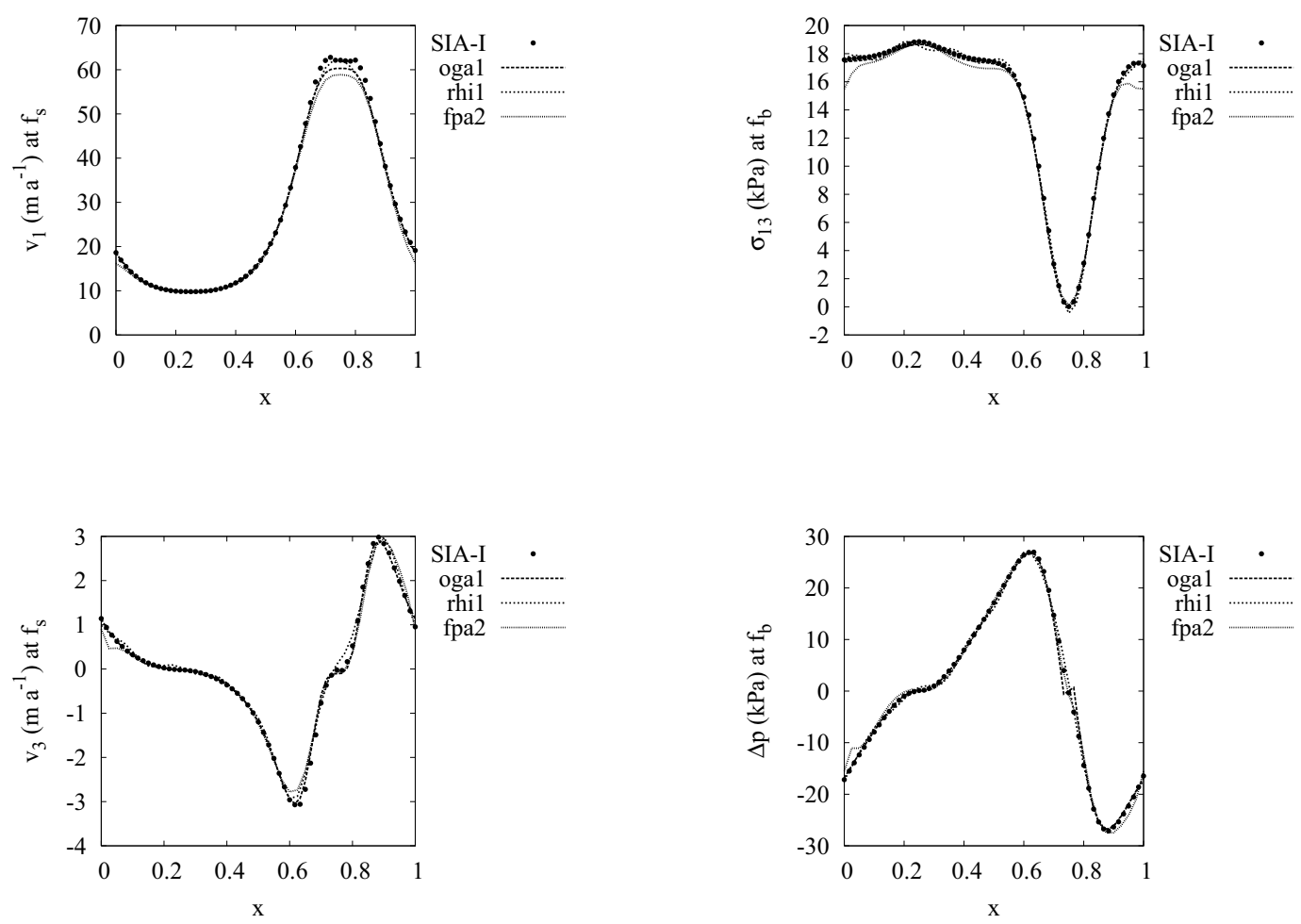

Fig. 6. Comparison of the surface velocity fields, $v_{1}$ and $v_{3}\left(\mathrm{ma}^{-1}\right)$, obtained by the SIA-I solver (black points) and the full-Stokes solvers oga1, rhi1 and fpa2 from the ISMIP-HOM C experiment, for the aspect ratio $\epsilon=\frac{1}{80}$. The displayed results are taken at an intersection of the scaled domain with the plane $y=0.25$.

With this modification, the SIA-I algorithm was tested using real data from the Antarctic region, considering, in addition, temperature-dependent viscosity. Without presenting any results, we only state that, for a reasonably smooth Dirichlet condition on velocity at the glacier bed, the performance of the SIA-I approach is comparable to the no-slip case. We intend to publish details of this result in a future paper.

To involve the sliding at the glacier bed, it is, however, necessary to change the Dirichlet boundary condition to the Newton boundary condition. Although we have not participated in the ISMIP-HOM C experiment, we are still able to make a few remarks on the SIA-I performance for this case, when basal sliding is concerned.

The problem is set up similarly to experiment $A$, but the driving effect is, instead of bed-geometry undulations, spatial inhomogeneities in the basal-friction coefficient. The upper and lower surfaces are both inclined planes given (in meters) by

$$
\begin{aligned}
& f_{\mathrm{s}}\left(x_{1}, x_{2}\right)=-x_{1} \tan \alpha, \quad \alpha=0.1^{\circ}, \\
& f_{\mathrm{b}}\left(x_{1}, x_{2}\right)=f_{\mathrm{s}}\left(x_{1}, x_{2}\right)-1000 .
\end{aligned}
$$

At the sides, periodic boundary conditions are again prescribed for the velocity field. At the glacier bed, the following sliding law is considered:

$$
\beta^{2} \vec{t} \cdot \vec{v}=\vec{t} \cdot \boldsymbol{\sigma} \cdot \vec{n},
$$

where $\vec{t}$ and $\vec{n}$ are the tangent and upward normal vectors to the glacier base, $f_{\mathrm{b}}$, respectively. The sliding coefficient is given by

$$
\beta^{2}\left(x_{1}, x_{2}\right)=1000+1000 \sin \left(\omega x_{1}\right) \sin \left(\omega x_{2}\right),
$$

with

$$
\omega=\frac{2 \pi}{[L]} .
$$

The Dirichlet condition on velocity, either in the form of no-slip or a prescribed velocity, is essential for the SIA-I algorithm since it allows a straightforward computation of the velocities by integration along the vertical coordinate. That is why the sliding law, Equation (67), has to be transformed to a Dirichlet-type condition for velocity. This can be done as follows. For $\beta(\cdot) \neq 0$, the stress field from the previous half-step can be used as

$$
\vec{t} \cdot \vec{v}^{0 k+\frac{1}{2}}=\frac{\vec{t} \cdot \sigma^{k+\frac{1}{2}} \cdot \vec{n}}{\beta^{2}} .
$$

The sliding velocity, $\vec{v}^{0 k+\frac{1}{2}}$, is then substituted in Equations (63-65) for $\vec{v}^{0}$. Evidently, this approach can only be applied to the region with $\beta \neq 0$ while, in the region where $\beta=0$, the algorithm fails. Such a situation occurs in the experiment $C$ setting, where $\beta=0$ at two points. To avoid the failure of the SIA-I approach, we add a small positive constant to $\beta$ and successively decrease it during the iterations.

The results are shown in Figures 6 and 7 . The plotted quantities are velocities $v_{1}, v_{3}$ at the upper surface (in $\mathrm{m} \mathrm{a}^{-1}$ ) and the stress component, $\boldsymbol{\sigma}_{13}$, and pressure difference $\Delta p=p-H \rho g$ at the bottom (in $\mathrm{kPa}$ ). As in the previous experiment, all these quantities are mapped onto the scaled domain $\langle 0,1\rangle \times\langle 0,1\rangle$ and the solutions are plotted at the cross-section with the plane $y=0.25$. For the comparison, we plot three full-Stokes solutions from ISMIP-HOM experiment $\mathrm{C}$, that is the models oga1, fpa2 and rhi1. We show the results for two aspect ratios, $\frac{1}{80}$ in Figure 6 , and $\frac{1}{20}$ in Figure 7 . All solutions are computed with a resolution of $31 \times 31 \times 31$, and are stopped after 200 iterations. The projection parameters are $\theta_{1}=0.2, \theta_{2}=0.02$ for aspect ratio $\epsilon=\frac{1}{80}$ and $\theta_{1}=0.1, \theta_{2}=0.01$ for aspect ratio $\epsilon=\frac{1}{20}$. 

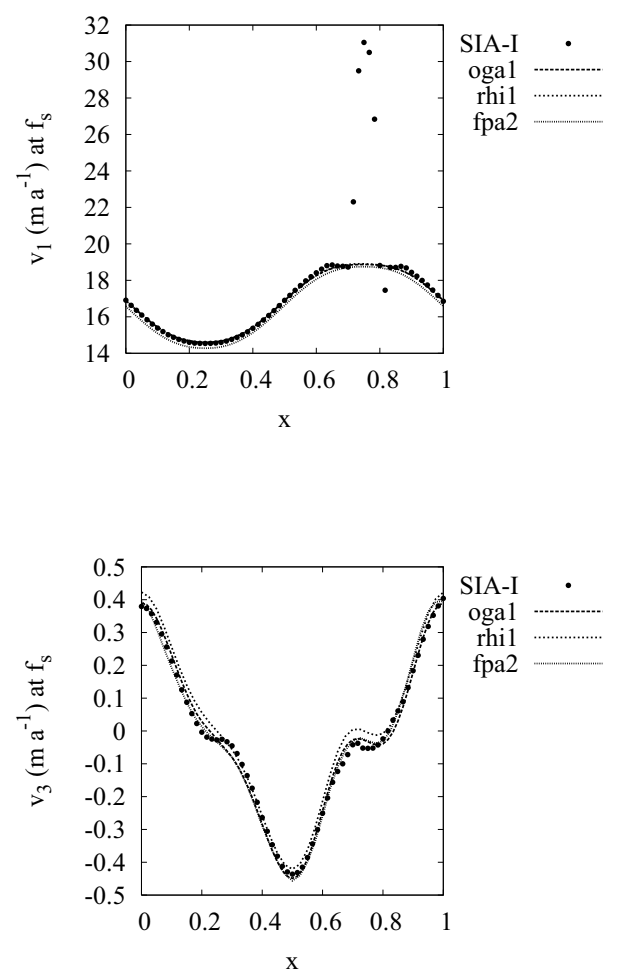

Fig. 7. As for Figure 6, but for $\epsilon=\frac{1}{20}$.

To compute each example takes 50 s of CPU time on an Intel Pentium 4 with $3.2 \mathrm{GHz}$.

Figures 6 and 7 show that, in accordance with our assumption, the SIA-I algorithm fails to compute the horizontal velocities correctly in the neighborhood of the point where the sliding friction coefficient $\beta=0$, that is at the point $(0.75,0.25)$ in our case. Moreover, the error increases with the increasing aspect ratio. However, the error is localized in a small region surrounding the point with $\beta=0$, and the stresses are well computed everywhere, even for a relatively large aspect ratio $\left(\frac{1}{20}\right)$. In general, we may conclude that the Newton boundary condition at the glacier base, i.e. the sliding law of the form in Equation (67), represents a restriction for the applicability of the SIA-I only in the cases when the region of a very small basal friction coefficient, $\beta$, is present.

\section{Numerical performance}

The essential feature of the presented SIA-I approach is its computational effectiveness. The algorithm is designed such that the time cost spent at each iterative step is similar to that required for the SIA approach. Using an Intel Pentium 4, 3.2 GHz computer, we have performed 50 iterations for the ISMIP-HOM A setting with $\epsilon=\frac{1}{80}$, which is a sufficient number of iterations for the SIA-I solution to converge to the full-Stokes solution. In Figure 8 we plot the total CPU time for SIA-I as a function of degrees of freedom, that is the number of computed velocity and stress components on a staggered grid. We can see that computational time increases linearly with increasing number of degrees of freedom.

Since our full-Stokes solver is not optimized for numerical performance, we consider CPU-time demands for computational runs of the professionally optimized finite-element solver Elmer (Gagliardini and Zwinger, 2008). For the current ISMIP-HOM A setting, the authors provide an analytical
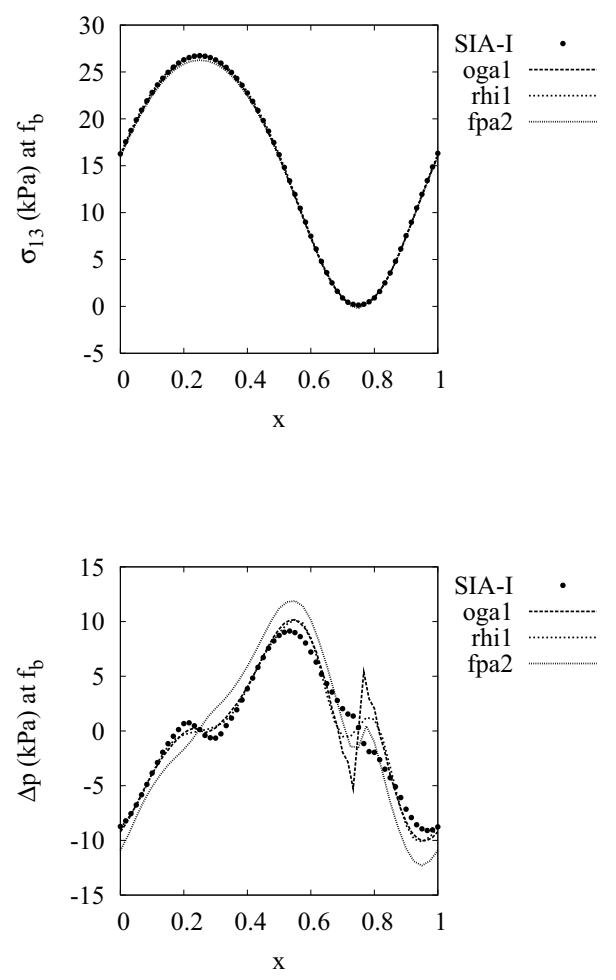

formula for CPU-time costs in seconds as a function of the number of degrees of freedom: $y=0.013 x^{1.11}$. If we make a similar estimate for the SIA-I solver, we obtain $y=0.00015 x$ (see Fig. 8).

\section{CONCLUSIONS}

The new iterative SIA-I algorithm presented in this paper is derived from the traditional SIA assumption of the smallness of the aspect ratio of the vertical/horizontal dimensions of a glacier. The algorithm represents an iterative extension of the SIA approach, and, in general, may provide a substantially improved solution of the Stokes flow problem. The key parameters controlling the performance of the SIA-I algorithm are the aspect ratio and projection parameters, $\theta_{1}$, $\theta_{2}$. For the model example taken from the ISMIP-HOM A experiment with $\epsilon \leq \frac{1}{10}$, the SIA-I algorithm converges if sufficiently small projection parameters are chosen. The case with $\epsilon=\frac{1}{10}$ is a threshold above which the SIA-I algorithm fails to converge, resulting in inaccurate and noisy results.

Besides its relative simplicity, the greatest advantage of the SIA-I algorithm is its fast computational speed, since it is designed such that the numerical computations consist of only numerical integration over the vertical coordinate and the differentiation of field quantities, similar to numerical operations performed within the SIA approach. Moreover, the computational demand grows only linearly with the number of degrees of freedom.

The performance of the SIA-I algorithm was also tested for the ISMIP-HOM experiment $C$ where a Newton-type sliding law is applied at the glacier base. The SIA-I approach requires the reformulation of the sliding law as a Dirichlet boundary condition for velocity that results in wrongly computed velocities in the regions of small sliding friction coefficient, 


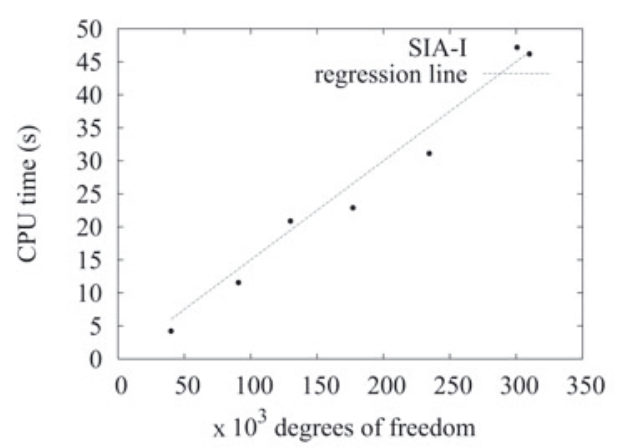

Fig. 8. CPU-time demands of the SIA-I algorithm as a function of degrees of freedom for the ISMIP-HOM A setting with $\epsilon=\frac{1}{80}$ and for 50 iterations, computed on Intel Pentium 4, 3.2GHz.

$\beta$. However, the errors in the velocities are localized in the vicinity of the region where $\beta=0$. The erroneous behavior of the SIA-I algorithm disappears with decreasing aspect ratio. For instance, in the case where $\epsilon=\frac{1}{80}$, the SIA-I converges to the exact solution everywhere in the solution domain.

\section{ACKNOWLEDGEMENTS}

This work was supported by the Center for Earth Dynamic Research and also by the Grant Agency of Charles University in Prague through grant No. UK/252217. The second author acknowledges support from the Grant Agency of the Czech Republic through grant No. 205 /06/0580. Both authors thank F. Pattyn, R. Greve and an anonymous reviewer for constructive comments and K. Fleming for English corrections.

\section{REFERENCES}

Blatter, H. 1995. Velocity and stress fields in grounded glaciers: a simple algorithm for including deviatoric stress gradients. J. Glaciol., 41(138), 333-344.

Gagliardini, O. and T. Zwinger. 2008. The ISMIP-HOM benchmark experiments performed using the Finite-Element code Elmer. Cryos. Discuss., 2(1), 75-109.

Granas, A. and J. Dugundji. 2003. Fixed point theory. New York, Springer-Verlag.

Greve, R. 1997. A continuum-mechanical formulation for shallow polythermal ice sheets. Philos. Trans. R. Soc. London, Ser. A, 355(1726), 921-974.

Hindmarsh, R.C.A. 2004. A numerical comparison of approximations to the Stokes equations used in ice sheet and glacier modeling. J. Geophys. Res., 109(F1), F01012. (10.1029/2003JF000065.)
Hutter, K. 1983. Theoretical glaciology; material science of ice and the mechanics of glaciers and ice sheets. Dordrecht, D. Reidel Publishing.

Le Meur, E., O. Gagliardini, T. Zwinger and J. Ruokolainen. 2004. Glacier flow modelling: a comparison of the Shallow Ice Approximation and the full-Stokes equation. C. R. Phys., 5(7), 709-722.

Paterson, W.S.B. 1981. The physics of glaciers. Second edition. Oxford, etc., Pergamon Press.

Pattyn, F. 2003. A new three-dimensional higher-order thermomechanical ice-sheet model: basic sensitivity, ice stream development, and ice flow across subglacial lakes. J. Geophys. Res., 108(B8), 2382.

Pattyn, F. and 20 others. 2008. Benchmark experiments for higherorder and full Stokes ice sheet models (ISMIP-HOM). Cryos. Discuss., 2(1), 111-151.

Press, W.H., S.A. Teukolsky, W.T. Vetterling and B.P. Flannery. 1992. Numerical recipes in FORTRAN: the art of scientific computing. Second edition. Cambridge, Cambridge University Press.

Price, S.F., E.D. Waddington and H. Conway. 2007. A fullstress, thermomechanical flow band model using the finite volume method. J. Geophys. Res., 112(F3), F03020. (10.1029/2006JF000724.)

Saito, F., A. Abe-Ouchi and H. Blatter. 2003. Effects of first-order stress gradients in an ice sheet evaluated by a three-dimensional thermomechanical coupled model. Ann. Glaciol., 37, 166-172.

Zwinger, T., R. Greve, O. Gagliardini, T. Shiraiwa and M. Lyly. 2007. A full Stokes-flow thermo-mechanical model for firn and ice applied to the Gorshkov crater glacier, Kamchatka. Ann. Glaciol., 45, 29-37.

\section{APPENDIX \\ THE FINITE-DIFFERENCE FULL-STOKES SOLVER}

To carry out the benchmarks against which the SIA-I solution could be checked, we developed a simple fullStokes solver. The governing equations (11-13), (14) and (24-31) are rewritten in stretched coordinates (see, e.g., Pattyn, 2003). The spatial derivatives are approximated by two-point symmetric differences and the resulting system of non-linear algebraic equations is solved on the staggered grid with two types of alternating nodes, first for the rheology equations and the equation of continuity, the others for the momentum balance equations. For a fixed viscosity, the linear system of equations is solved by a PARDISO (Parallel Sparse Direct Linear Solver) routine (http://www.intel.com), and the viscosity is iteratively updated by the convex combination of the previous and updated velocity fields. The convergence is checked by inspecting the evolution of the maximal difference between two successively computed velocity fields. 